\title{
Study of the role of epididymal $\alpha$-glucosidase in the fertility of male rats by the administration of the enzyme inhibitor castanospermine
}

\author{
C. H. Yeung and T. G. Cooper \\ Institute of Reproductive Medicine of the University, Steinfurterstrasse 107, D-48149 Münster, Germany
}

\begin{abstract}
The activity of epididymal $\alpha$-glucosidase in adult rats was rapidly suppressed to histochemically undetectable levels within 2 days by the continuous release of the enzyme inhibitor castanospermine via a peritoneal osmotic pump at a rate of $100-200 \mathrm{nmol} \mathrm{h}^{-1}$. It was established that mating activities overnight depleted $72 \%$ of the spermatozoa in the distal cauda, which was replenished in 2 days, and that fertility began to decline 3 weeks after efferent duct ligation. Male rats of proven mating proficiency and fertility were treated with castanospermine, or buffered saline as control, for up to 30 days and enzyme inhibition was confirmed at the end of treatment by histochemistry. Fertility was normal at the first mating test on day 7 , significantly decreased at the second mating on day 9, but recovered in a stepwise manner at subsequent matings on days 12 and 14. Delaying the third mating until day 25 did not sustain the transient subfertility. However, prolonging sperm storage in the distal cauda epididymides and preventing replenishment with freshly matured spermatozoa, by efferent duct ligation for 14 days performed on day 15 during castanospermine administration, caused a decrease in fertility and a change in the kinematics of epididymal spermatozoa of the castanospermine-treated group. In control rats, binding of epididymal spermatozoa to Vicia faba, a lectin specific for glucose and glucosamine, and mannose and mannosamine residues, decreased from the proximal caput to the distal corpus coincident with the increase in $\alpha$-glucosidase activity on the epithelial brush border. Lectin binding then increased in the cauda where enzyme activity was absent. However, castanospermine treatment did not significantly alter this binding profile. The findings suggest that epididymal $\alpha$-glucosidase does not play a crucial role in the development of sperm fertilizing capacity, but may be involved in the preparation of spermatozoa for storage.
\end{abstract}

\section{Introduction}

The activity of $\alpha$-glucosidase in human seminal plasma has been used as a marker of epididymal function (Casano et al., 1987; Cooper et al., 1988; Guerin et al., 1986) and is increasingly used in clinical diagnosis (WHO Manual, 1992). Its activity is correlated with sperm concentration and motility in the ejaculate and testosterone concentration in serum below $30 \mathrm{nmol} \mathrm{I}{ }^{-1}$ (Cooper et al., 1988). The neutral form of the enzyme originates almost exclusively from the epididymis (Tremlay et al., 1982; Paquin et al., 1984; Cooper et al. 1990) and its histochemical activity is localized on the brush border of the human epididymal epithelium, reaching maximal intensity in the corpus and cauda epididymides (Yeung ef al., 1990) as well as in the proximal vas deferens (C. H. Yeung, unpublished). However, the physiological role of epididymal $\alpha$-glucosidase remains obscure. Among the laboratory animals investigated, the rat is the only species displaying enzyme activity on the epididymal brush border, activity increases from the caput to a maximum in the corpus but is absent from the distal cauda epididymidis (Yeung et al., 1990).
Whereas the acidic lysosomal $\alpha$-glucosidase hydrolyses glucose residues, the other intracellular glucosidases (the neutral isoenzymes associated with the endoplasmic reticulum and Golgi apparatus) are responsible for the initiation of oligosaccharide processing in the synthesis of $N$-linked glycoproteins (Spiro et al., 1989). A precursor of lysosomal $\alpha$-glucosidase was found to be secreted and concentrated on the epithelial brush border of the human intestine (Fransen et al., 1988) and proximal renal tubules (Elferink et al., 1989) and, unlike other lysosomal enzymes, this apical secretion does not involve the mannose-6-phosphate receptor (Klumperman et al., 1992). However, the function of this secretory isoenzyme is unknown.

The natural substrate for epididymal glucosidase has not been established, but possibilities include glycoproteins free in the luminal fluid or on the sperm surface. Epididymal glycoproteins have been shown to regulate the fertilizing ability of rat epididymal spermatozoa in vitro (Cuasnicu et al., 1984). Modification of the carbohydrate groupings on the sperm surface has been identified in many species as the spermatozoa pass through the epididymis (Cooper, 1986). For the rat, changes in surface glucose and mannose residues have been demonstrated by changes in agglutination with lectins or 
fluorescence (Lewin et al., 1979; Olson and Danzo, 1981). Such changes could be brought about by the combined actions of glucosyltransferases and glucosidases, both of which are present in epididymal luminal fluid (Hamilton, 1980; Hamilton and Gould, 1982). Mannosidase, which is also an asparaginelinked glycoprotein processing enzyme, has been found in the rat epididymis in the Golgi membrane, acting on tissue specific endogenous substrates (Skudlarek et al., 1991). Although there is no evidence for secretion, it has been identified on the rat sperm membrane (Tulsiani et al., 1989). An alteration in epididymal glycoproteins has been shown in rats fed the mannosidase inhibitor swainsonine, but the effects on sperm function or fertility were not investigated (Tulsiani $e$ t al., 1989). $\beta$-Galactosidase has been detected in the rat epididymal fluid and on spermatozoa (Skudlarek et al., 1993), but its function is also unclear.

Castanospermine is a selective inhibitor of glucosidase and it does not affect other lysosomal glycosidases including mannosidase, galactosidase, fucosidase, acetylhexosaminidase and glucuronidase (Saul et al., 1983). It has been shown to inhibit seminal (Cooper et al., 1990) and epididymal $\alpha$-glucosidase (Yeung et al., 1990). In the present study, castanospermine was administered to rats to investigate whether epididymal $\alpha$-glucosidase plays a role in fertility regulation, either through an action on the maturation or storage of epididymal spermatozoa. The route of administration, effective dosage and time course of enzyme inhibition were first established. The extent of depletion of the mature sperm store by mating and the time for refilling by abstinence were estimated, as was the maximal storage time for maintenance of the fertility capacity of mature spermatozoa. The results from the study reported here and from other studies on the transit time of spermatozoa in the epididymis, as well as on spontaneous ejaculation, formed the basis of the design of experimental protocols and mating schedules aimed at testing the effect of castanospermine on maturation and storage of epididymal spermatozoa.

\section{Materials and Methods}

\section{Animals}

Adult male Sprague-Dawley rats (360-400 g initial body mass, Charles River Deutschland, Sulzfeld) were housed singly during the experiment. Female rats $(220-380 \mathrm{~g})$ were caged either in groups of two or three during monitoring of oestrous cycles or singly after a mating was confirmed. All rats were kept on a schedule of $12 \mathrm{~h}$ light:12 $\mathrm{h}$ dark and had access to rat chow and water ad libitum.

\section{Recruitment and handling of male rats for fertility studies}

Only male rats with proven mating proficiency were used. Starting 3 weeks before control or drug treatment, each male rat was placed with an oviduct-ligated female at pro-oestrus overnight and the mating was confirmed by the presence of spermatozoa in the vaginal smear the next morning. Up to four such mating tests were allowed for each male at intervals of 3-7 days and only those achieving three positive matings with not more than one failure were recruited into the study.
Pretreatment fertility was monitored by placing an intact female at pro-oestrus into the cage of the male the day before drug administration began.

\section{Recruitment and handling of female rats for fertility studies and} for the mating training of male rats

The oviducts of rats used for establishing mating proficiency in males were ligated under halothane (Hoescht AG, Frankfurt am Main) anaesthesia via bilateral incisions and after a recovery period of 2 weeks, the oestrous cycle was monitored by daily vaginal smears made in the afternoon (as described by Oberländer et al., 1994). For both intact and oviduct-ligated females, only those with regular cycles were used. When a mating test was required, a female showing a pro-oestrous smear was introduced into the cage of the male and the vaginal smear was examined the following morning for the presence of spermatozoa.

Inseminated intact females were separated and killed 12 days later by asphyxiation using $\mathrm{CO}_{2}$. The number of implantations and corpora lutea from each side were counted and the fertility rate $(100 \% \times$ number of implantations/number of corpora lutea) of each female was expressed as the mean of left and right sides. Inseminated oviduct-ligated rats were used again after two weeks or longer.

\section{Dose and time course of castanospermine effect}

Castanospermine, supplied as a generous gift by E. H. W. Bohme (Marion Merrell Dow, Cincinnati, $\mathrm{OH}$ ), was dissolved in PBS at 9, 20, 100 or $200 \mathrm{mmol} \mathrm{l}^{-1}$ and used to fill mini-osmotic pumps (Alzet pumps; Charles River Deutschland, Sulzfeld) which released the drug at a rate of $1 \mu \mathrm{h} \mathrm{h}^{-1}$. Similar pumps were filled with PBS for controls. Each filled pump was soaked for $4 \mathrm{~h}$ in PBS for equilibration of release rate, then placed in the peritoneal cavity through a central abdominal incision made under halothane anaesthesia. In some pilot experiments, the pump was sutured onto the wall of the inguinal canal and the outlet was connected with tubing, the tip of which was secured by ligation to the gubernaculum, such that the content would be released directly into the scrotum. Rats were killed after 1, 2, 3 or 7 days. Epididymides and kidneys were frozen in liquid nitrogen and stored at $-80^{\circ} \mathrm{C}$ for the measurement of glucosidase activities.

\section{Measurement of a-glucosidase activity within epididymal tubules and in the kidney}

Cryosections of tissues were cut and fixed as described by Yeung et al. (1990). Enzyme activity on the brush border of the epididymal tubule and kidney cortex was measured using 5-bromo-4-chloro-3-indolyl-D-glucopyranoside as substrate at $\mathrm{pH} 6.5$, and quantified by including increasing concentrations of the inhibitor castanospermine in the incubation medium, as described by Yeung et al. (1990). Distal corpus epididymidis and kidney sections of non-treated male rats were included in each incubation as negative (renal medulla) and positive (renal cortex) controls as well as intra-assay quality control (coefficient of variation was $14 \pm 5 \%$ for corpus and $1.1 \pm 0.6 \%$ for 
kidney). A glucosidase index was used to express enzyme activity, which was taken as the lowest concentration of castanospermine (in $\mu \mathrm{mol} 1^{-1}$ ) required to inhibit completely the formation of the coloured enzyme product in the tissue section.

\section{Measurement of epididymal sperm motility}

The detailed procedure described by Yeung ef al. (1992) was used. In brief, the animal was anaesthetized with urethane (Sigma, Deisenhofen) and a portion of the luminal content from defined regions of the epididymis was carefully released into the incubation medium. Sperm motility was examined immediately and after incubation for $3 \mathrm{~h}$ at $34^{\circ} \mathrm{C}$ in $5 \% \mathrm{CO}_{2}$. Sperm movement in $40 \mu \mathrm{m}$ deep siliconized chambers was recorded and analysed using the Hamilton-Thorn computer system. Kinematic parameters measured for $3 \mathrm{~s}$ at 7 frames $\mathrm{s}^{-1}$ included curvilinear velocity (VCL, calculated from the path constructed by joining consecutive track points), averaged path velocity (VAP), straight line velocity (VSL, from the distance from first to last track points), linearity (LIN, 100\% $\times \mathrm{VSL} /$ VCL), straightness (STR, $100 \% \times \mathrm{VAP} / \mathrm{VCL}$ ) and amplitude of lateral displacement (ALH). From each sample, the median value of 100 or more sperm tracks for each movement parameter was obtained. Percentage motility was estimated by counting motile and immotile spermatozoa from the video monitor.

\section{Lectin-binding capacity of epididymal spermatozoa}

Agglutination in the presence of lectin was taken as a measure of sperm surface carbohydrate groups. Since no lectins are available that bind solely to D-glucose residues, lectins were chosen that bound glucose in addition to D-mannose. Of three lectins examined (Concanavalin A, Vicia sativa and Vicia faba) the latter was chosen because of the nature of the agglutination observed with caudal spermatozoa. In contrast to the others, Vicia faba caused tail-tail agglutination which was clearly evident as long strands of spermatozoa. This was also clearly quite different from the wheat sheaf type of mixed agglutination characteristic of the clumps of effete spermatozoa often found in the epididymis (Cooper and Hamilton, 1977).

Spermatozoa were released from the rat caput, corpus and cauda epididymidis by mincing in Dulbecco's PBS, gravitational separation of tissue debris and collection of the supernatant. Because of the fragile nature of rat spermatozoa (Jones, 1986) and the desire to monitor plasma membrane residues, rather than intracellular components, spermatozoa were not washed. Spermatozoa were diluted to $30 \times 10^{6} \mathrm{ml}^{-1}$ as judged from a nephelometric standard curve read against a PBS blank at $405 \mathrm{~nm}$. The same number of spermatozoa (300 000 in $10 \mu \mathrm{l}$ aliquots) were added to $100 \mu \mathrm{l}$ PBS (control value) and lectin (100 $\mu \mathrm{g} \mathrm{ml}^{-1}$ and doubling dilutions thereof) in flat-bottom 96 well plates which were then shaken on a microtitre plate shaker at room temperature for 20-30 min. Each well was scrutinized under an inverse microscope (Zeiss IM) at $\times 3.2-32$ for evidence of agglutination. Heavy agglutination was obvious at $\times 3.2$ as clumping of cells; higher magnification was used to determine whether adjacent cells were associated (close adherence of adjacent flagella giving the appearance of abnormally long sperm tails). Wells were classified as demonstrating agglutination $(+)$ or not $(-)$; where only few cells exhibited agglutination, the grading \pm was given and the next dilution was recorded as the concentration of lectin at which no agglutination was visible.

Confirmation of the lectin specificity was indicated in preliminary experiments in which no agglutination was observed when the lectin-containing PBS also contained 0.1 mol $\alpha$-methylglucosidase $1^{-1}$ or $\alpha$-methylmannoside, but not $\beta$-methylglucoside. Providing that equal numbers of spermatozoa are added to the wells, the smaller amount of lectin required to cause agglutination should reflect a greater number of lectin-binding sites on the sperm surface (Sarkar et al., 1991).

\section{Depletion and refilling of epididymal sperm store by mating activities}

Adult virgin male rats (300-400 g body mass) were caged singly until they were placed with one or two females at pro-oestrus. The next morning, after the confirmation of mating by the presence of spermatozoa in the vaginal smear, the male rat was killed and each epididymis was dissected into four segments: the proximal half up to the isthmus of the corpus, distal corpus, proximal cauda up to the flexture, and the distal cauda excluding any vas deferens. Each segment was weighed and minced in $1 \mathrm{ml}$ saline in a Petri dish. The dish contents were transferred to a capped tube and placed horizontally onto a multiwell plate shaker for $3 \mathrm{~min}$. The mixture was homogenized with three strokes of a glass Elvehjem homogenizer. From each homogenate, dilutions were made separately from two aliquots and the number of sperm heads in each dilution was counted twice in a Fuchs-Rosenthal haemocytometer and means taken from the quadruplicate counts.

The refilling of the epididymal sperm store was investigated by counting epididymal spermatozoa in another group of rats 2 days after each male had mated with two females on the same night. Naive males were used as controls.

\section{Fertility of rats with ligated efferent ducts and motility of stored spermatozoa}

Adult male rats $(360-480 \mathrm{~g})$ were anaesthetized with halothane. Each testis was exposed through a central abdominal incision and the efferent ducts were separated from vascular and fatty tissue and ligated with 5/0 silk suture thread before it was returned to the scrotum. Sham-operated animals, in which efferent ducts were separated but no ligature was placed, were used as controls. Each rat was mated once with one female at $2,3,4$ or 6 weeks after the operation and the fertility of each mated female was examined as described above. After mating was confirmed, the motility of spermatozoa recovered from the distal cauda was analysed. Testis and epididymal masses were obtained and serum testosterone was measured by radioimmunoassay as described by Chandolia et al. (1991). Control rats were examined 2 weeks after the operation. 
Effect of castanospermine administration on the fertility and sperm motility of male rats

On day 0 , the day after mating with an intact female (termed day -1), a mini-osmotic pump filled with castanospermine was placed in the peritoneal cavity of the male rat as described above. The administration regimen and subsequent mating schedule were designed differently in the following three experiments to differentiate any effect of glucosidase inhibition on already matured or maturing spermatozoa, or on sperm storage.

Experiment 1. Castanospermine was administered at $400 \mathrm{mmol} \mathrm{l}^{-1}$ at $0.46 \mu \mathrm{h} \mathrm{h}^{-1}$ (14 day pump) and each treated rat mated on days $7,9,12$ and 14 . The rationale was to examine the effect on already matured spermatozoa (present in the cauda epididymidis before treatment) and ejaculated in the first mating (day 7) following treatment. The second mating (day 9) would involve mostly spermatozoa matured during treatment and residual spermatozoa matured before treatment. The fertility of spermatozoa freshly matured during treatment would be evaluated by the last two matings.

Experiment 2. Castanospermine was administered at $45 \mathrm{mmol} \mathrm{l}{ }^{-1}$ at $2.5 \mu \mathrm{h} \mathrm{h}^{-1}$ ( 28 day pump) and each rat mated on days $7,9,25,27$ and 30 . The rationale for matings on days 7 and 9 was as above; on days 25 and 27 it was for the examination of spermatozoa matured and stored during drug treatment; and the mating on day 30 was for the testing of spermatozoa freshly matured during treatment.

Experiment 3. Castanospermine was administered at $400 \mathrm{mmol} \mathrm{l}^{-1}$ at $0.48 \mu \mathrm{L} \mathrm{h}^{-1}$ (14 day pump) with matings on days 7 and 9. On day 15, efferent duct ligation was performed in both the control and the treated groups as described above and another 14 day pump containing castanospermine was provided. The last mating was on day 29 for the examination of stored spermatozoa only, without any freshly matured cells.

For each group, similar pumps filled with PBS were given to similarly recruited control rats and the same mating schedule applied as to the treatment group. Occasionally no rat at pro-oestrus was available or to be expected on the scheduled mating day; the mating was then performed 1 day before or after. Rarely did the male not mate when a female was placed in its cage. In these cases, mating then took place the following day with another pro-oestrous rat. The fertility rate of the male was obtained from each inseminated female as described above. After the final mating, epididymal sperm motility was videorecorded and the sperm kinematics were analysed. The lectinbinding capacity of spermatozoa from various epididymal regions was estimated. Liver, kidney, testis, epididymis and seminal vesicle (longitudinally cut and fluid expressed) were weighed. Blood from the tail was collected in two heparinized capillary tubes and centrifuged for $15 \mathrm{~min}$ at $13000 \mathrm{~g}$ at room temperature for haematocrit measurement. The effectiveness of castanospermine administration was checked by snap freezing the kidneys and distal corpus epididymides in liquid nitrogen and storage at $-80^{\circ} \mathrm{C}$ for histochemical quantification of $\alpha$-glucosidase activity.

\section{Statistical analyses}

Data were analysed using ANOVA by Statgraphics software (STSC Inc., Rockville, MD). Angular transformation (asin $\sqrt{ }(x / 100)$ ) was applied to values in percentages, including percentage motility, LIN, STR and fertility rates, before analy. sis and mean values were retransformed when given in the text. Student's $t$ test was used when there was only one treated group versus a control group. When there were more groups of data, such as those obtained from various periods of efferent duct ligation and fertility data on various days of treatment within the same experimental design, differences between data groups were analysed using Duncan's multiple-range test, and were considered statistically significant with $P<0.05$.

\section{Results}

\section{Dose dependence and time course of the effect of castanospermine}

There was a dose-dependent inhibition of $\alpha$-glucosidase activity in the epididymis by $20-200 \mathrm{mmol}$ castanospermine $1^{-1}$ administered via mini-osmotic pumps (Table 1 ). However, attempts to localize the effect to the epididymis by releasing castanospermine directly into one side of the scrotum did not prevent simultaneous inhibition of enzyme activity in the kidney. However, release into the peritoneal cavity (200 mmol castanospermine $\mathrm{l}^{-1}$ at $1 \mu \mathrm{h} \mathrm{h}^{-1}$ ) achieved the same marked epididymal inhibition in 1 day, resulting in non-detectable histochemical enzyme activity in 2 days. The same dose $\left(400 \mathrm{mmol} \mathrm{l} \mathrm{l}^{-1}\right.$ at about $\left.0.5 \mu \mathrm{h} \mathrm{h}^{-1}\right)$ was adopted for subsequent fertility studies using pumps designed for administration for 14 days whereby total inhibition was effective for up to 16 days (Table 1 ). In view of the minimal dose required to achieve complete inhibition within 2 days $\left(100 \mathrm{mmol} \mathrm{l}^{-1}\right.$ at $1 \mu \mathrm{h} \mathrm{h}^{-1}$; i.e. $100 \mathrm{nmol} \mathrm{h}^{-1}$ ), 28 -day pumps were also used to administer castanospermine at $112 \mathrm{nmol} \mathrm{h}^{-1}$

\section{Depletion and refilling of epididymal sperm store by mating activities}

In sexually inactive rats, there was a marked increase in sperm content from the distal corpus to the proximal cauda, and then a slight decrease in the distal cauda (Fig. 1). After mating with one female, sperm content in the distal cauda decreased to $28 \%$ of control values and there was no further decrease in rats mated with two females on the same night. (This indicated either that the total number of emissions was the same with one or two mating partners, or that the distal cauda was emptied to the maximal extent by mating for one night, regardless of the number of mated females.) Whereas sperm content in the proximal cauda decreased to a much lesser extent if at all (to about $70 \%$ of control values, not significant), that in the distal corpus increased by about $50 \%(P<0.001)$. (This may reflect enhancement of sperm transport from upstream after sexual activity, or a reflux of proximal cauda spermatozoa owing to contractile activity during emission.) Two days after mating, sperm distribution in the epididymis returned to the control pattern and the sperm content of the three regions recovered to near control values (Fig. 1). 
Table 1. $\alpha$-Glucosidase activity indices in rats treated with increasing concentrations of castanospermine continuously released from osmotic pumps into one side of the scrotum (i.s.) or intraperitoneally (i.p.), starting from day 0

\begin{tabular}{|c|c|c|c|c|c|}
\hline \multirow[b]{2}{*}{ Castanospermine concentration } & \multirow[b]{2}{*}{ Day } & \multirow[b]{2}{*}{$n$} & \multicolumn{2}{|c|}{ Corpus epididymidis } & \multirow[b]{2}{*}{ Kidney } \\
\hline & & & Ipsilateral & Contralateral & \\
\hline Untreated & & 6 & $44 \pm 5$ & & $140 \pm 17$ \\
\hline \multicolumn{6}{|l|}{ at $1 \mu \mathrm{h} \mathrm{h}^{-1}$ (7-day pumps) } \\
\hline Control (i.s.) & 10 & 3 & $40 \pm 0$ & $47 \pm 12$ & $157 \pm 5$ \\
\hline \multirow[t]{2}{*}{$20 \mathrm{mmol} \mathrm{l}^{-1}$ (i.s.) } & 2 & $I$ & 10 & 20 & 5 \\
\hline & 7 & I & 2 & 20 & 5 \\
\hline \multirow[t]{2}{*}{$100 \mathrm{mmol} \mathrm{l}^{-1}$ (i.s.) } & 2 & 1 & 0 & 3 & 2 \\
\hline & 7 & 1 & 0 & 1 & 2 \\
\hline \multirow[t]{4}{*}{$200 \mathrm{mmol} \mathrm{l}^{-1}$ (i.s.) } & 1 & 3 & $1 \pm 1$ & $3 \pm 2$ & $2 \pm 1$ \\
\hline & 2 & 3 & $0 \pm 0$ & $0 \pm 0$ & 1 \\
\hline & 10 & 3 & $0 \pm 0$ & $0.5 \pm 1$ & $1 \pm 1$ \\
\hline & 11 & 2 & 0.5 & 0.5 & 2 \\
\hline \multirow[t]{2}{*}{$200 \mathrm{mmol} \mathrm{l}^{-1}$ (i.p.) } & 1 & 3 & $2^{a}$ & & 0 \\
\hline & 2 & 2 & $0^{a}$ & & 0 \\
\hline \multicolumn{6}{|l|}{ at $0.5 \mu \mathrm{h}^{-1}$ (I4-day pumps) } \\
\hline Control (i.p.) & 15 & 6 & $50 \pm 11$ & & $155 \pm 5$ \\
\hline $400 \mathrm{mM}$ (i.p.) & 16 & 6 & $0 \pm 0$ & & $0 \pm 0$ \\
\hline
\end{tabular}

Values are means \pm sD for $n$ rats.

${ }^{a}$ Both sides.

Fig. 1. Sperm contents of the distal regions of the rat epididymidis ( $\square$ : distal corpus; : proximal cauda; $\mathbf{Q}$ : distal cauda) from animals that were not mated (control, $n=6$ ), mated with one female $(n=8$ ) or two females $(n=4)$, or mated with one female and the number of spermatozoa determined 2 days later $(n=5)$. Values are means + SEM. *Significantly different $(P<0.05)$ from the same region of the controls.

Effect of epididymal storage on the fertility and motility of cauda spermatozoa studied by efferent duct ligation in otherwise untreated rats

After efferent duct ligation, testis mass decreased gradually to reach a steady value of $50 \%$ by 3 weeks, although there was no decline in serum testosterone throughout the 6 weeks (Table 2). Epididymal mass decreased to a steady value of about $70 \%$ of that of sham-operated rats by 2 weeks. Whereas the fertility rate was normal 2 weeks after the operation, it fell in two of the four rats after 3 weeks, although all females mated were pregnant. This had further declined to complete infertility by 6 weeks. The effect on motility followed a different profile. Although percentage motility was already affected 2 weeks after ligation, the kinematic characteristics of the motile cells were indistinguishable from the controls, as with fertility. However, motile spermatozoa were still present in three rats which were completely infertile by 4 or 6 weeks. The decline in fertility was parallel to the decrease in straight-line velocity and increase in lateral displacement (ALH), although most differences from control values did not reach statistically significant levels (Table 2).

Effect of castanospermine administration on the fertility and sperm motility of male rats

Administration of castanospermine using an osmotic pump did not affect body growth or mass of testes, epididymides, seminal vesicles, liver and kidney (data not shown). There was no difference in serum testosterone or haematocrit values between control and treated animals. Epididymal $\alpha$-glucosidase was suppressed to histochemically non-detectable levels in all 16 treated animals by the end of the treatment, except in three rats from Expt 2 in which very weak activities were still measurable (enzyme activity-index $1.2 \pm 0.5$ versus control value of $56 \pm 4$ ).

Experiment 1: effect on already matured or freshly matured spermatozoa. All six animals receiving control pumps sustained high fertility rates, as shown by the serial mating tests throughout the two weeks. In the castanospermine group, there was large variation; two rats remained fully fertile, one became totally infertile in two mating tests from day 9 on but 
Table 2. Reproductive parameters of normal adult rats after efferent duct ligation

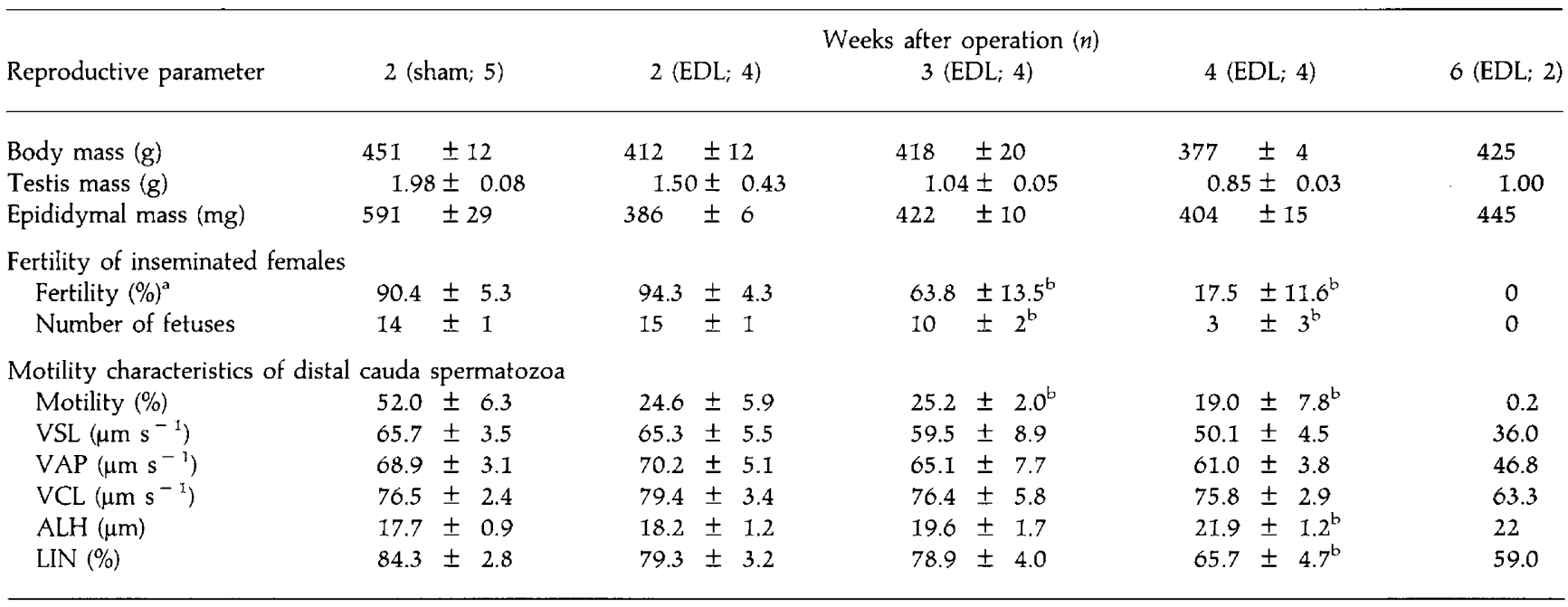

Values are means \pm SEM.

${ }^{a}$ Number of fetuses/number of corpora lutea $\times 100 \%$.

'Significantly different from the sham operated animals $(P<0.05)$.

EDL: efferent duct ligation; VSL: straight line velocity; VAP: averaged path velocity; VCL: curvilinear velocity; ALH: amplitude of lateral displacement; LIN: linearity.

recovered in the last mating on day 16; two others showed a decline in fertility on day 9 which returned to the control value at the end of treatment; and one showed a gradual decline without recovery. Both the fertility rate and the number of fetuses in the females mated on day 9 were significantly lower than both their own pretreatment values and control values of the same treatment day (Fig. 2). The fertility rate was still slightly reduced at day 12 , but totally recovered at the next and last mating.

Spermatozoa obtained from the distal corpus, proximal and distal cauda epididymides were indistinguishable from those of control animals in percentage motility and all other kinematic parameters analysed (data not shown, but similar to those reported in Yeung et al., 1992).

Experiment 2: effect on already matured, freshly matured and stored spermatozoa. All control rats exhibited consistently high fertility rates over the six matings of the experiment (Fig. 3). Castanospermine drastically affected two of the five treated rats. One of these was completely infertile on day 9 but fully recovered in the next mating 2 weeks later. The other was completely infertile for the two matings after administration of the pump (days 7 and 9) and produced only one fetus in the female inseminated on day 25 . Thereafter, fertility returned to normal.

There was no difference between control and treated animals in motility and kinematics of spermatozoa from the distal corpus and distal cauda either immediately after release into medium or after incubation for $3 \mathrm{~h}$ (data not shown).

Experiment 3: effect on already matured, freshly matured, or segregated stored spermatozoa. In contrast to the first two experiments, the mean fertility rate of the treated group on day

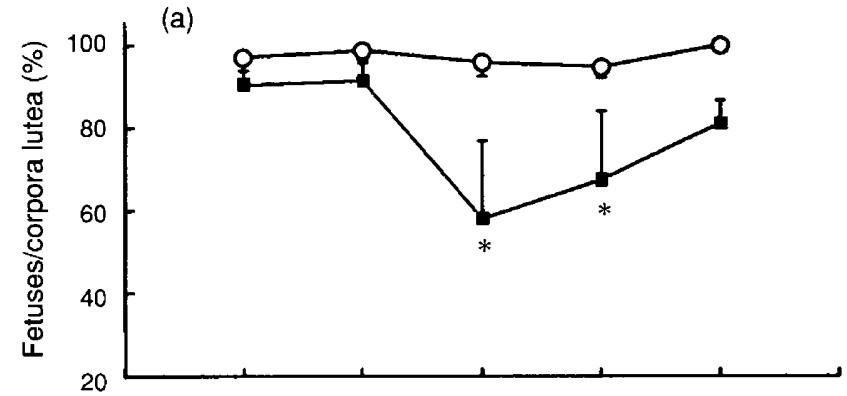

(b)

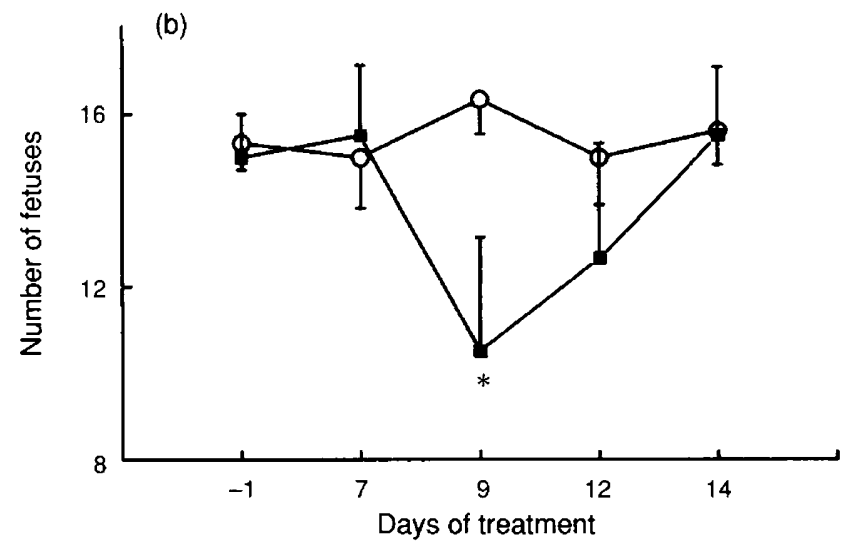

Fig. 2. Effect of continuous administration of castanospermine for 14 days (Expt I) in (O) control and ( $\boldsymbol{0}$ ) treated animals on male rat fertility expressed as (a) the number of fetuses from each mated female per corpora lutea and (b) as number of fetuses. Mean $\pm \operatorname{SEM}(n=6)$. * Significantly different from control values for the same period of treatment $(P<0.05)$

9 was not significantly lower than the control value. In control rats, full fertility was maintained 2 weeks after ligation of the efferent duct. In the castanospermine-treated group, fertility 


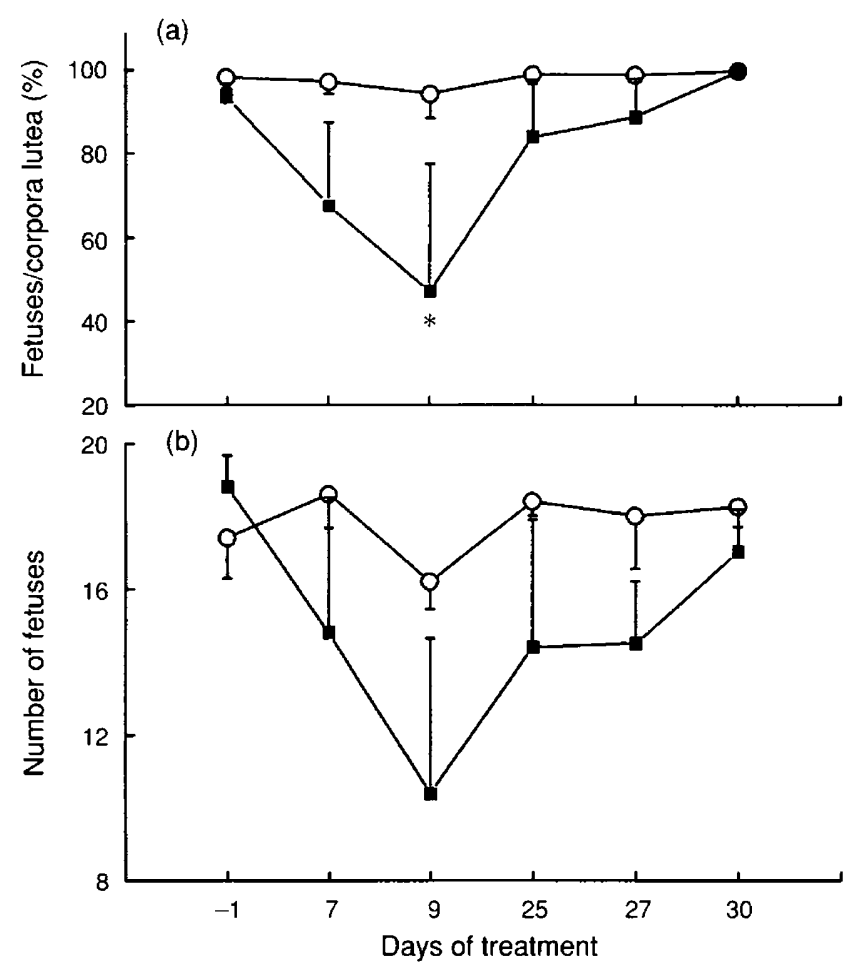

Fig. 3. Effect of continuous administration of castanospermine for 30 days (Expt 2) in (O) control and (ש) treated animals on male rat fertility expressed as (a) the number of fetuses from each mated female per corpora lutea and (b) as number of fetuses. Mean $\pm \operatorname{SEM}(n=5)$. * Significantly different from control values for the same period of treatment $(P<0.05)$.

was significantly decreased, although completely absent in only one of five animals (Fig. 4).

When immediately released into medium, cauda spermatozoa from both groups of rats showed statistically similar kinematics, although treated rats exhibited slightly lower straight-line velocity (VSL) and larger lateral displacement $(\mathrm{ALH})$. After $3 \mathrm{~h}$ incubation, control spermatozoa maintained their curvilinear velocity while ALH increased, resulting in a decline in VSL. Treated spermatozoa showed different changes upon incubation, with a significant decrease in ALH (Fig. 5).

\section{Effect of epididymal a-glucosidase inhibition on lectin-induced} sperm agglutination

In spermatozoa from control rats, the minimum concentration of lectin that induced sperm agglutination increased gradually from the proximal caput to reach a peak in the distal corpus, followed by a decrease in the cauda regions (Fig. 6). In rats treated for a month with castanospermine, such a profile was still observed in spermatozoa obtained along the length of the epididymis. However, the minimal lectin concentration was higher than the control value in each region from the proximal caput to the distal corpus and lower in the proximal and distal cauda, where concentrations were declining. However, none of the intra-region comparisons revealed a statistically significant difference. In Expt 3, spermatozoa retained in the cauda epididymidis after efferent duct ligation again showed
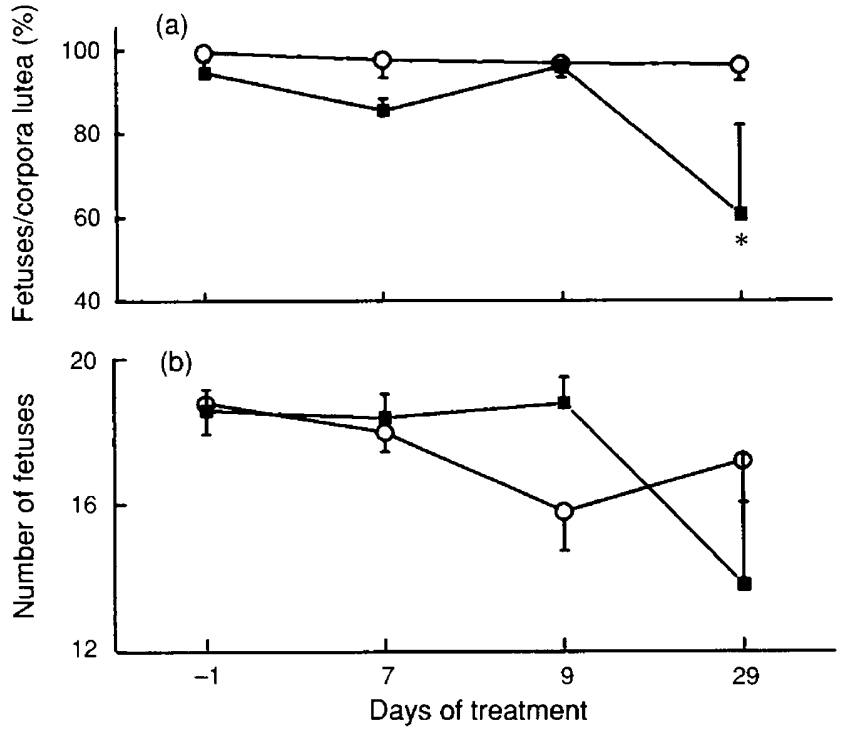

Fig. 4. Effect of continuous administration of castanospermine for 29 days with efferent duct ligation for both control and treated groups on day 15 (Expt 3) in (O) control and ( $\boldsymbol{\square}$ ) treated animals on male rat fertility expressed as (a) the number of fetuses from each mated female per corpora lutea and (b) as number of fetuses. Mean $\pm \operatorname{SEM}(n=5)$. *Significantly different from control values for the same period of treatment $(P<0.05)$.

agglutination at a lower, albeit non-significant, lectin concentration when treated with castanospermine for 29 days (8.7. \pm 4.1 versus $11.9 \pm 5.4 \mu \mathrm{g} \mathrm{ml}^{-1}$ for controls).

\section{Discussion}

Complete abolition of the histochemical activity of epididymal $\alpha$-glucosidase by the administration of castanospermine via mini-osmotic pumps was achieved without apparent side effects on the general health of the rats, although simultaneous inhibition of enzyme activity in the kidney was demonstrated. The use of a recruitment regimen to ensure the mating proficiency and fertility of the experimental rats provided control groups with consistently high fertility baselines which improved the sensitivity of the fertility tests. It also eliminated the positive bias caused by serial mating using naive animals (Zenick and Goeden, 1988).

The number of epididymal spermatozoa stored in the distal cauda epididymidis was decreased by $75 \%$ when measured at mid-morning after the male was placed with a female overnight. One rat ejaculate contains about $20 \%$ (Bedford, 1990) or $60 \%$ (Ratnasooriya and Wadsworth, 1987) of the spermatozoa stored in the cauda. Nevertheless, mating with two instead of one female in the same night did not further deplete the sperm store. The present study showed that this store was refilled to near previous levels in 2 days. Mating following this initial emptying would thus involve spermatozoa previously held in the proximal cauda.

The first mating performed by the experimental rats after the start of drug treatment was on day 7. Their fertility was not statistically different from that of the controls in all three 


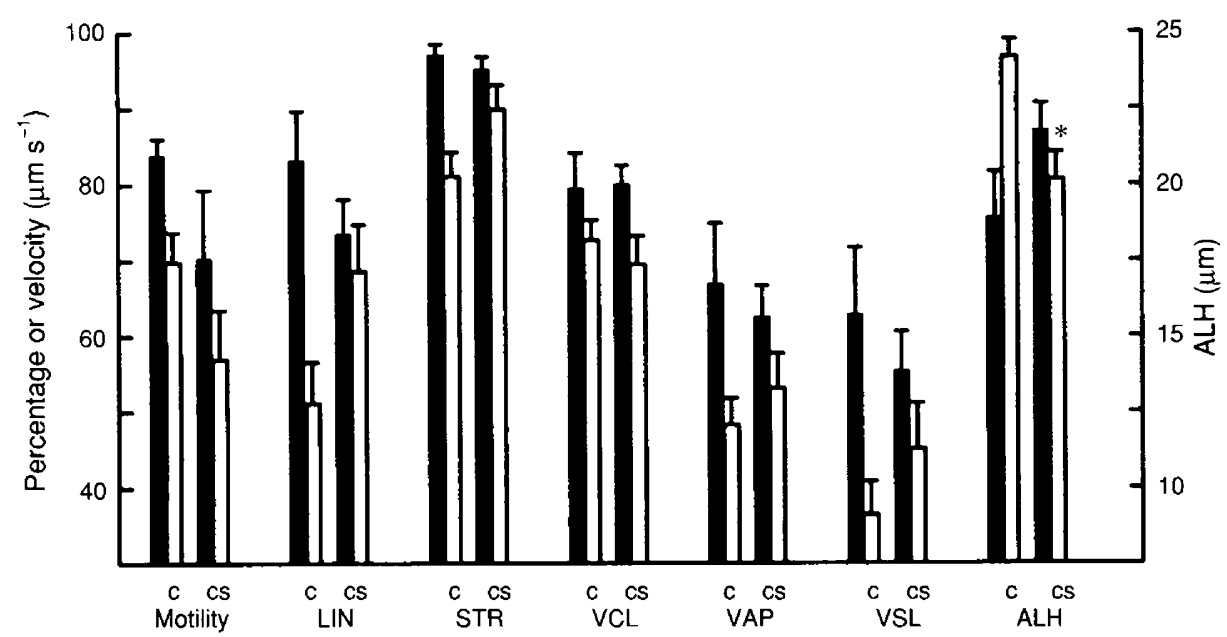

Fig. 5. Motility parameters of rat cauda epididymal spermatozoa obtained after 29 days of continuous administration of castanospermine, with efferent duct ligation on day 15 (Expt 3), examined immediately $(\mathrm{O}, \mathbf{h})$ or after incubation $(3 \mathrm{~h}, \Xi)$. For each parameter, values from control $(\mathrm{C})$ and castanospermine treated (CS) groups are shown in pairs. Means $+\operatorname{SEM}(n=5)$. *Significantly different $(P<0.05)$ from control values for the same incubation time. Motility, linearity (LIN) and straightness of path (STR) are as percentage (left ordinate); curvilinear velocity (VCL); average path velocity (VAP) and straight line velocity (VSL) are in $\mu \mathrm{m} \mathrm{s}^{-1}$ (left ordinate), amplitude of lateral displacement (ALH) is in $\mu \mathrm{m}$ (right ordinate).

experiments, suggesting that there was no effect on spermatozoa that had already matured before the administration of castanospermine. After this mating, the distal cauda epididymidis would be refilled by spermatozoa, most of which had travelled through an epididymal lumen devoid of $\alpha$-glucosidase activity. The sperm transit time through the entire rat epididymis is 8.4 days (Robb et al., 1978) or 7-10 days in freely mating animals (Martan and Risley, 1963) and $\alpha$-glucosidase activity is weak on the epithelial brush border of the caput epididymidis but increases markedly in the corpus regions (Yeung et al., 1990). It can therefore be postulated that most spermatozoa ejaculated in the second mating test during treatment (mating on day 9) had matured in the absence of, or in the presence of sparse, glucosidase activity, and that some would have been stored in the cauda a few days after maturation. From the mating on day 9 in both Expts 1 and 2, fertility was lower than in the controls. However, variation between animals was large and the effect was not repeated in Expt 3. Nevertheless, when data from all three experiments were pooled for matings on days $-1,7$ and 9 , a decrease reaching statistical significance was found for day 9 when compared with day 9 of the control or pretreatment values $(69.8 \pm 11.6$ versus $95.7 \pm 1.7$ and $92.8 \pm 1.8 \%$, respectively; retransformed mean \pm SEM).

If the effect on day 9 were a result of castanospermine affecting sperm maturation, a more pronounced effect would be expected later in the treatment. However, this possibility was excluded by the return of normal fertility in subsequent matings during drug administration. Our working hypothesis was that spermatozoa freshly matured in the absence of a-glucosidase can be fertile, but an interaction with the enzyme during maturation is required to maintain their fertilizing ability during storage in the epididymis. This view is consistent with the abrupt loss of glucosidase activity from the epithelial

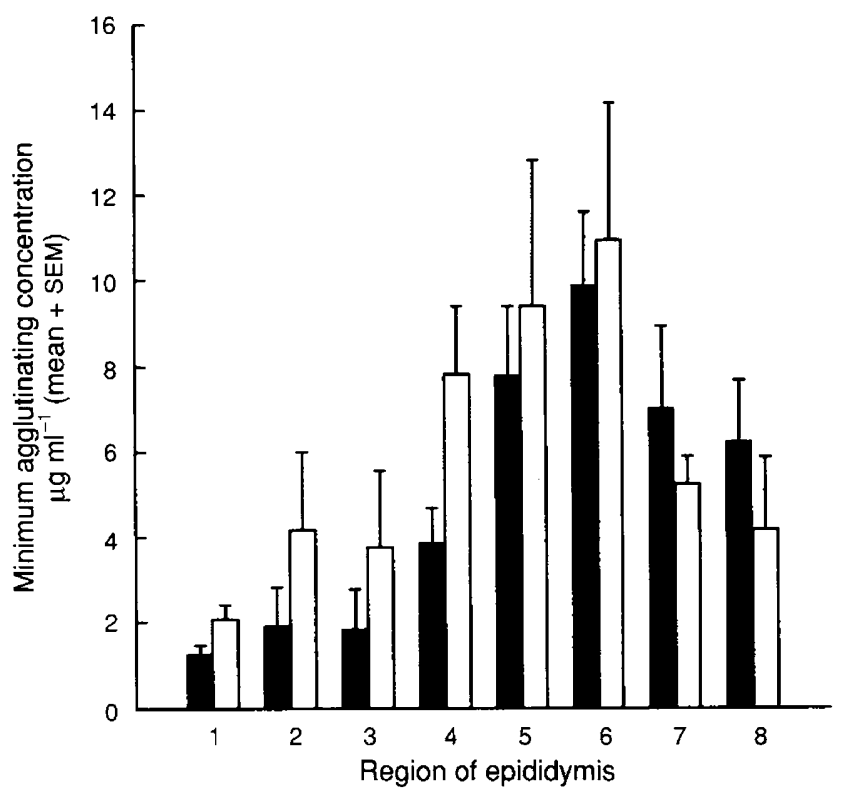

Fig. 6. Profiles of lectin (Vicia faba) binding, reciprocally expressed as the minimum concentrations of lectin required to induce agglutination of spermatozoa obtained along the length of the epididymis: regions (1) proximal caput, (2) mid caput, (3) distal caput, (4) proximal corpus, (5) mid corpus, (6) distal corpus, (7) proximal cauda and (8) distal cauda in (a) control and $(\square)$ castanospermine treated animals (5 rats for 30 days and 1 for 14 days) (mean + SEM; $n=6)$.

brush border coinciding with the spatial division of the sperm storage and maturation functions of the epididymis. Spermatozoa in subsequent matings (on day 12 and 14) would not have 
been stored for long because of the frequent mating trials and there would be no manifestation of the castanospermine effect.

This hypothesis was tested by not allowing castanosperminetreated rats to mate after two mating sessions (days 7 and 9) in an attempt to retain the spermatozoa, all of which would have matured and been stored in the absence of glucosidase, for 16 days before permitting the next mating. In Expt 2, however, female rats inseminated after this interval did not show a significant decrease in fertility. This finding may not preclude the hypothesis, in view of the reported phenomenon of spontaneous ejaculation in rats, which would have rendered ineffective the prolongation of the storage of cauda spermatozoa by avoiding mating activity. Assuming the average production of two spontaneous ejaculates per day, each containing $10 \%$ of the spermatozoa in a normal copulatory ejaculate (Ågmo, 1976), it takes only 5 days for one turnover of the sperm store under normal non-mating circumstances. However, such spontaneous ejaculation is suppressed for 4-9 days after mating with a female (Beach, 1975). This effect was overcome by ligating the efferent duct in Expt 3 during castanospermine administration after the first two matings, to prevent the continuous flow of freshly matured spermatozoa into the cauda. This should then contain only spermatozoa that had matured and been stored without epididymal glucosidase activity. There was a slight, yet statistically significant, decrease in the mean fertility rate despite the large individual variation in the response. This effect was accompanied by a decrease in the amplitude of the lateral movement of the motile spermatozoa upon incubation, which may suggest a defect in hyperactivation or capacitation.

The endogenous substrate of $\alpha$-glucosidase in the epididymis is not known. The amount of sperm surface glucose or glucosamine and mannose or mannosamine residues decreased gradually from the proximal caput to the distal corpus as reflected by the increasing concentration of the lectin Vicia faba required to induce sperm agglutination. This decrease parallels the increasing enzyme distribution pattern. Where the epididymal brush border enzyme activity is lost in the cauda (Yeung et al., 1990), lectin binding increased. However, this binding profile persisted in castanospermine treated rats, indicating that the relationship between epididymal glucosidase activity and sperm surface glucose residues is not simple. The limited specificity of Vicia faba means that the decline in agglutinability of spermatozoa upon epididymal passage may well reflect loss of surface mannose rather than of glucose residues. In a study in which the total inhibition of glucosidase can achieve only $50 \%$ suppression of $N$-linked oligosaccharide processing, deglucosylation blockade is found to be circumvented by an endo-D-mannosidase (Moore and Spiro, 1990). Whether such alternative enzyme activity occurs in the castanospermine treated epididymis is not known.

The present findings suggest that epididymal $\alpha$-glucosidase does not play a crucial role in the development of the fertilizing capacity of spermatozoa, although it may be involved in facilitating sperm storage.

The authors thank E. Möllmann for technical assistance and E. H. W. Bohme of Marion Merrell Dow (Cincinnati, OH) for the gift of $\alpha$-glucosidase. This work was supported by the Deutsche Forschungsgemeinschaft grant no. Ni130/11-3.

\section{References}

Agmo A (1976) The number of spermatozoa in spontaneous ejaculates of rats Journal of Reproduction and Fertility 48 405-407

Beach FA (1975) Variables affecting "spontaneous" seminal emission in rats Physiology and Behavior 15 91-95

Bedford JM (1990) Sperm dynamics in the epididymis. In Gamete Physiology, pp 53-67 Eds RA Asch, JP Balmaceda and I Johnston. Serono Symposia, Norwell

Casano R, Orlando C, Caldini AL, Barni T, Natali A and Serio M (1987) Simultaneous measurement of seminal L-carnitine, alpha-1,4-glucosidase and glycerylphosphorylcholine in azoospermic and oligospermic patients Fertility and Sterility $47 \quad 324-328$

Chandolia RK, Weinbauer GF, Behre HM, Simoni M and Nieschlag E (1991) Comparative effects of chronic administration of the non-steroidal antiandrogens Flutamide and Casodex on the reproductive system of the adult rat Acta Endocrinologica 105 547-550

Cooper TG (1986) The Epididymis, Sperm Maturation and Ferfilisation Springer Verlag, Heidelberg

Cooper TG and Hamilton DW (1977) Observations on destruction of spermatozoa in the cauda epididymidis and proximal vas deferens of nonseasonal male mammals American Journal of Anatomy 149 93-110

Cooper TG, Yeung CH, Nashan D and Nieschlag E (1988) Epididymal markers in male infertility Journal of Andrology 9 91-101

Cooper TG, Yeung CH, Nashan D, Jockenhoevel F and Nieschlag E (1990) Improvement in the assessment of human epididymal function by the use of inhibitors on the assay of $\alpha$-glucosidase in seminal plasma International Journal of Andrology 13 297-305

Cuasnicu PS, Gonzales Echeverria F, Piazza AD, Cameo MS and Blaquier A (1984) Antibodies against epididymal glycoproteins block fertilizing ability in rats Journal of Reproduction and Fertility $72 \quad 467-471$

Elferink RPJO, Fransen JAM, Klumperman J, Ginsel LA and Tager JM (1989) Secretion of a precursor form of lysosomal $\alpha$-glucosidase from the brush border of human kidney proximal tubule cells European Journal of Cell Biology $50299-303$

Fransen JAM, Ginsel LA, Cambier PH, Klumperman J, Elferink RPJO and Tager JM (1988) Immunocytochemical demonstration of the lysosomal enzyme $\alpha$-glucosidase in the brush border of human intestinal epithelial cells European Journal of Cell Biology 47 72-80

Guerin JF, Benal H, Rolletz J, Soucher C and Czyba JC (1986) Alpha-glucosidase as a specific epididymal enzyme marker - its validity for the etiologic diagnosis of azoospermia Journal of Andrology 7 156-162

Hamilton DW (1980) UDP-galactose: $N$-acetylglucosamine galactosyltransferase in fluids from rat rete testis and epididymis Biology of Reproduction $\mathbf{2 3}$ 377-385

Hamilton DW and Gould RP (1982) Preliminary observations on enzymatic galactosylation of glycoproteins in the surface of rat caput epididymal spermatozoa International Journal of Andrology Supplement 5 73-80

Jones R (1986) Preparation of a plasma membrane-rich fraction from rat spermatozoa Journal of Reproduction and Fertility 77 435-449

Klumperman J, Fransen JAM, Tager JM and Ginsel LA (1992) The cationindependent mannose 6-phosphate receptor is not involved in the polarized secretion of lysosomal $\alpha$-glucosidase from Caca- 2 cells European Journal of Cell Biology 57 147-154

Lewin LM, Weissenberg R, Sobel JS, Marcus Z and Nebel L (1979) Differences in concanavalin A-FITC binding to rat spermatozoa during epididymal maturation and capacitation Archives of Andrology 2 279-281

Martan J and Risley PL (1963) The epididymis of mated and unmated rats Journal of Morphology 113 1-15

Moore SEH and Spiro R (1990) Demonstration that Golgi endo- $\alpha-D-$ mannosidase provides a glucosidase-independent pathway for the formation of complex $N$-linked oligosaccharides of glycoproteins Journal of Biological Chemistry 265 13104-13112

Oberländer G, Yeung CH and Cooper TG (1994) Induction of reversible infertility in male rats by oral ornidazole and its effects on sperm motility and epididymal secretions Journal of Reproduction and Fertility 100 551-559

Olson GE and Danzo BJ (1981) Surface changes in rat spermatozoa during epididymal transit Biology of Reproduction 24 431-443 
Paquin R, Chapdelaine P, Dube JY and Tremblay RR (1984) Similar biochemical properties of human seminal plasma and epididymal a-1,4-glucosidase Journal of Andrology 5 277-282

Ratnasooriya WD and Wadsworth RM (1987) Effect of mating on sperm distribution in the reproductive tract of the male rat Gamete Research 17 261-266

Robb GW, Amann RP and Killina GJ (1978) Daily sperm production and epididymal sperm reserves of pubertal and adult rats journal of Reproduction and Fertility 54 103-107

Sarkar M, Majumder GC and Chatterjee T (1991) Goat sperm membrane lectin-binding sites of sperm surface and lectin affinity chromatography of the mature sperm membrane antigens Biochimica et Biophysica Acta 1070 198-204

Saul R, Chambers JP, Molyneux RJ and Elbein AD (1983) Castanospermine, a tetrahydroxylated alkaloid that inhibits $\beta$-glucosidase and B-glucocerebrosidase Archives in Biochemistry and Biophysics 221 593-597

Skudlarek MD, Orgebin-Crist M-C and Tulsiani DRP (1991) Asparagine-linked glycoprotein biosynthesis in rat epididymis. Presence of mannosidase Il-like enzyme Biochemical Journal 277 213-221

Skudlarek MD, Tulsiani DRP, Nagdas SK and Orgebin-Crist M-C (1993) $\beta$-D-galactosidase of rat spermatozoa: subcellular distribution, substrate specificity, and molecular changes during epididymal maturation Biology of Reproduction 49 204-213
Spiro RC, Casteel HE, Laufer DM, Reifeld RA and Harper IR (1989) Posttranslational addition of chondroitin sulfate glycosaminoglycans. Role of $N$-linked oligosaccharide addition, trimming, and processing Journal of Biological Chemistry 264 1779-1786

Tremblay RR, Chapdelaine P, Roy R and Thabet M (1982) Correlation between เ-carnitine and alpha-1,4-glucosidase activity in the semen of normal, infertile and vasectomized men Infertility 5 61-70

Tulsiani DRP, Skudlarek MD and Orgebin-Crist M-C (1989) Novel $\alpha$-Dmannosidase of rat sperm plasma membranes: characterization and potential role in sperm-egg interactions Journal of Cell Biology 109 1257-1267

WHO (1992) Laboratory Manual for the Examination of Human Semen and Sperm-Cervical Mucus Interaction (3rd Edn). Cambridge University Press, Cambridge, UK

Yeung CH, Cooper TG and Senge T (1990) Histochemical localization and quantification of $a$-glucosidase in the epididymis of men and laboratory animals Biology of Reproduction 42 669-676

Yeung CH, Oberländer G and Cooper TG (1992) Characterization of the motility of rat spermatozoa by computer-aided objective measurement journal of Reproduction and Fertility 96 427-441

Zenick H and Goeden H (1988) Evaluation of copulatory behavior and sperm in rats: role in reproductive risk assessment. In Physiology and Toxicology of Male Reproduction, pp 179-201 Ed. JC Lamb and PMD Foster. Academic Press, London 